\title{
ANESTHETIC CONCERN IN MUCOPOLYSACHROIDOSIS
}

\author{
N. Girimurugan ${ }^{1}$, V. Santhosh², Remadevi R ${ }^{3}$, S. Yogeshwaran ${ }^{4}$
}

\section{HOW TO CITE THIS ARTICLE:}

N. Girimurugan, V. Santhosh, Remadevi R, S. Yogeshwaran. "Anesthetic Concern in Mucopolysachroidosis". Journal of Evolution of Medical and Dental Sciences 2014; Vol. 3, Issue 43, September 11; Page: 10652-10655, DOI: $10.14260 /$ jemds/2014/3387

INTRODUCTION: Mucopolysaccharidoses (MPS) are a group of progressive hereditary disorders of connective tissue metabolism in which lysosomal enzyme deficiency leads to intralysosomal deposition of mucopolysaccharides or glycosaminoglycans in the airway, cornea, brain, heart, liver, spleen, bones, ligaments, blood vessels, skin producing various symptoms.[1,2]

CASE HISTORY: An 8 year old female child clinically diagnosed as mucoplysaccharoisdosis (MPS) Type 1 (Hurlers syndrome) presented with noisy breathing and recurrent respiratory tract infection, was posted for adenostonsillectomy. Patient had a history of poor mentation and feeding problems. On examination child had a large head, large tongue, coarse facial features, and also had hepatosplenomegaly.

Routine lab investigations were normal and echocardiography showed normal study. The intra and postoperative complication were explained to the relatives and informed consent was obtained for adenotonsillectomy under General anesthesia. Difficult airway trolley was kept ready.

Patient was pre medicated with oral Midazolam.5mg/kg 1 hour prior to surgery, Glycopyrolate $0.2 \mathrm{mg}$ I.V was given. After pre oxygenation for 5 minutes, Fentanyl $2 \mu \mathrm{g} / \mathrm{kg}$ I.V, child was induced with Propofol $2 \mathrm{mg} / \mathrm{kg}$ I.V and Suxamethonium $1.5 \mathrm{mg} / \mathrm{kg}$ I.V was given after checking for adequate mask ventilation. Trial laryngoscopy was done it reveals Cormack-Lehane Grade-1, patient was intubated nasotracheally through right nostril after Oxymetazoline $0.05 \%$ drops and adequate lubrications.

Anesthesia was maintained with N20: 02 4:2 lit, Sevoflurane and Atracurium. The intraoperative course was uneventful and at the end of the procedure, after checking for adequate hemostasis, patient was reversed with glycopyrolate and neostigmine. Throat suction was done under direct vision. Patient was extubated after adequate swallowing reflex and obeying commands.

Patient shifted to post-operative ward after observing for 2 hours in recovery, 6 hours later, child developed continuous nasal bleeding, which was initially managed conservatively and nasal packing was done, since bleeding was continuous, planned for emergency re-exploration.

Preoperative resuscitation was done with isotonic crystalloid $20 \mathrm{ml} / \mathrm{kg}$. Haemoglobin and coagulation variables were be checked. Blood and blood products arranged.

During re-exploration, patient has to be considered as full stomach, previous anesthesia chart was reviewed, patient was hemodynamically stable and was induced with Propofol $1 \mathrm{mg} / \mathrm{kg} \mathrm{I.} \mathrm{V}$ Suxamethonium $(1 \mathrm{mg} / \mathrm{kg})$ I. V. On laryngoscopy epiglottis appeared edematous, then child was intubated orally with 5 size cuffed endotracheal tube.

Tonsillar fossa appeared clear and on nasal endoscopy revealed mucosal bleed on inferior turbinate which was cauterized nasal packing was done, after the procedure child was reversed and extubated. Child was not comfortable with the nasal packing; child was irritable in postoperative period in spite of adequate analgesia with injection Fentanyl $2 \mu \mathrm{g} / \mathrm{kg}$ I.V and Diclofenac suppository. 
DISCUSSION: The management of MPS is a challenge for the anesthesiologist especially in cases that require general anesthesia, rapid induction early emergence in children with delayed metabolism. The anesthetic risk of MPS patients must be considered high for many reasons, including the airway abnormalities, pulmonary pre- disposition, cardiac and neurological involvement.[3]

Operative risk is higher in Type I, II, IV and VI Types (Overall mortality rate in 20\% particularly, MPS I H is considered to the "Worst airway management problems a pediatric anesthesiologist could deal with" because of this, when possible general anesthesia should be avoided and when necessary, administered only by experienced anesthesiologist.[4]

\section{The management algorithm of anesthesia, MPS patients deals with $\mathbf{3}$ main concerns:}

1. Difficult intubation.

2. Chronic Pulmonary Disease.

3. Dangerous neck manipulations cervical instability.

The choice of anesthetic technique is the point of interest in MPS patients. General anesthesia is dangerous in MPS subjects and when possible local anesthesia with peripheral blocks should be preferred. ${ }^{[5]}$ Administering regional nerve block is difficult in MPS patient as most of them are mentally challenged. In order to reduce anesthetic risk, it is a common practice in MPS patients to do multiple procedures in a single anesthetic sitting.

Osthus et al[6] claimed that to reduce anesthetic risk diagnostic and surgical procedures should be combined. Airway abnormalities are consequences of morphological differences in MPS which leads to obstruction and difficult intubation resulting in possible CICV situations.[5]

MPS patients present commonly with ENT problems in particular Otitis media (30\%), hearing loss (75\%) adenotonsillar hypertrophy (75\%) frequent upper airway infections (75\%) and obstructive sleep apnea syndrome (45\%) and fifty percent of patients requiring surgical therapy.

Generally, oral intubations should be preferred due to adenoid \& tonsil dimensions, besides nasal mucosa weakness.[7] Moores et al reported seven children with MPS after stem cell transplantation undergoing anesthetic procedures in which conventional intubations was difficult in $72 \%$ \& failed in $29 \%\left[{ }^{[6]}\right.$.These patients are likely to have complications during recovery and immediate postoperative period such as apnea, bronchospasm, cyanosis and respiratory failure.[8]

In our case, we took all precautions expecting difficult airway. Nasal intubation was done as surgeon preferred it for tonsillectomy. We faced problems during extubation due to nasal congestion and edema. Six hours postoperatively patient developed nasal bleeding and was taken for second surgery, there was difficulty in mask ventilation and due to airway edema and nasal packing, and we needed a smaller size tube for intubation.

After cauterization of nasal bleeding, nasal packing was done which was difficult to tolerate by the patient during recovery. In spite of the anticipated postoperative airway obstruction we were forced to give opioid analgesic to keep the child calm and tolerate the nasal pack.

As the child was mentally challenged and had poor cognition, post operatively managing the child with nasal packing was a challenge. Even though we did not face intubation difficulty in both surgeries, there were challenges in managing the child post operatively complicated with nasal bleeding and second surgery. 
CONCLUSION: The MPS patients possess a major challenge to the anesthetist.The approach to the child planned for adenotonsillectomy and re-exploration in postoperative period requires a comprehensive awareness of multiple, sometimes conflicting concerns, and challenges the anaesthetist to balance risks with reality. We conclude that in MPS patients oral intubation may be preferred over nasal intubation as the disease process causes thinning of nasal mucosa which may cause nasal bleeding.

\section{REFERENCES:}

1. Moores C, Rogers J. G, Mckenzie I. M, Brown T. C. K. "Anaesthesia for children with mucopolysaccharidoses."Anaesthesia and Intensive Care, 1996; 24: 459-63.

2. Diaz JH, Belani KG. Perioperative management of children with Mucopolysaccharidosis. Anesth Analg, 1993; 77: 1261-70.

3. Walker R. W. M, Darowski M, Morris P, Wrait J E. "Anaesthesia and mucopolysaccharidoses. A review of airway problems in children" Anaesthesia, 1994; 49: 1078-84.

4. V. Valayannopoulos, F. A. Wijburg, "Therapy for the mucopolysaccharidoses, " Rheumatology, 2011; 50:49-59 supplement 5.

5. Tobias J. D. "Anesthetic care for the child with Morquio syndrome: general versus regional anesthesia," Journal of Clinical Anesthesia, 1999; 11: 242-46.

6. Osthaus W. A, THarendza, L. H. Witt et al., "Paediatric airway management in mucopolysaccharidosis 1: a retrospective case review," European Journal of Anaesthesiology, 2012; 29:204-7.

7. Bready L, S Noorily, D Dillman, Anestesiologia. Processo Decisionale, Elsevier, 2009.

8. Sjogren P, T. Pedersen, H. Steinmetz, "Mucopolysaccharidoses and anaesthetic risks," Acta Anaesthesiologica Scandinavica, 1987; 31: 214-18.

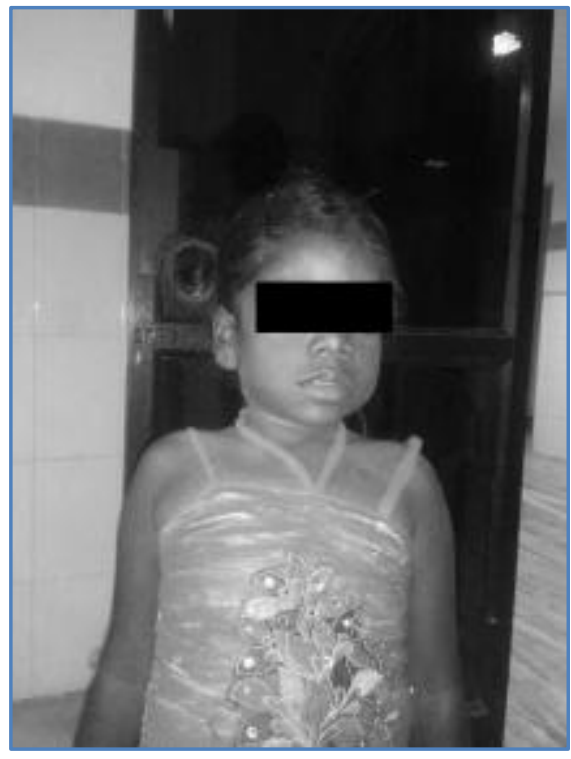

Figure 1

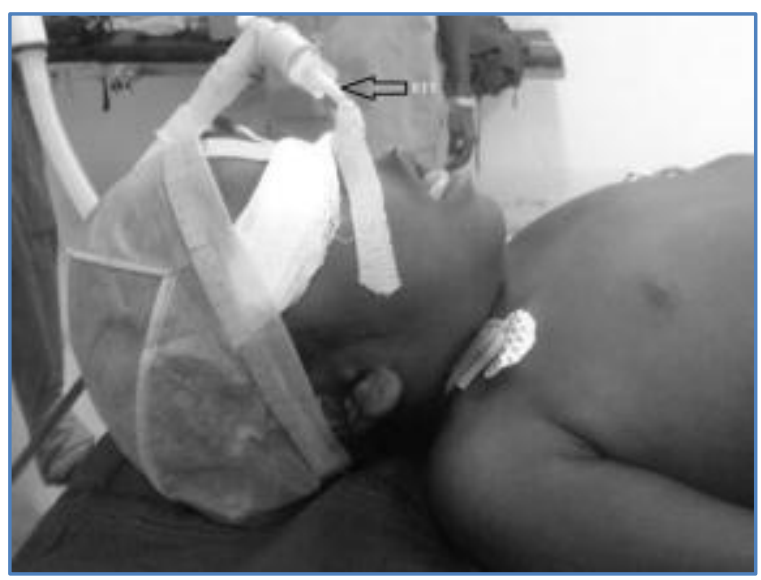

Figure 2 


\section{CASE REPORT}

\section{AUTHORS:}

1. N. Girimurugan

2. V. Santhosh

3. Remadevi R.

4. S. Yogeshwaran

\section{PARTICULARS OF CONTRIBUTORS:}

1. Post Graduate Student, Department of Anaesthesiology, Sri Manakula Vinayagar Medical College, Pondicherry.

2. Assistant Professor, Department of Anaesthesiology, Sri Manakula Vinayagar Medical College, Pondicherry.

3. Assistant Professor, Department of Anaesthesiology, Sri Manakula Vinayagar Medical College, Pondicherry.
4. Post Graduate Student, Department of Anaesthesiology, Sri Manakula Vinayagar Medical College, Pondicherry.

\section{NAME ADDRESS EMAIL ID OF THE CORRESPONDING AUTHOR:}

Dr. Remadevi R,

Type-V-A-13,

Jipmer Campus,

Pondicherry-605006.

Email: remadevi70@gmail.com

Date of Submission: 28/08/2014.

Date of Peer Review: 30/08/2014.

Date of Acceptance: 06/09/2014.

Date of Publishing: 09/09/2014. 